\section{Optimized Vector for Conditional Gene Targeting in Mouse Embryonic Stem Cells}

BioTechniques 34:1136-1140 (June 2003)

Gene targeting of the mouse genome has been widely used to create mouse mutants for exploring the biology of mammals and for better understanding human disorders in which the respective genes are functionally affected. With the advent of the mouse genome sequence, genetic manipulation of mice and the development of models for human diseases will be greatly facilitated (1). The loss-offunction approach by selectively inactivating specific (parts of) genes by homologous recombination in embryonic stem (ES) cells has been used extensively and successfully. However, it bears the risk that deletion of genes indispensable for embryonic development will lead to embryonic lethality, thus precluding the functional analysis of such genes in adult mice. The use of the bacteriophage $\mathrm{P} 1$-derived Cre/ loxP and yeast-derived Flp/frt systems in mouse genetics finally overcame these difficulties and allowed the study of the function of genes in vivo in a given organ or at a specific time during the life of a mouse (2-4). The establishment of mouse lines with conditional alleles is, however, more elaborate and time-consuming than the generation of conventional knockout mice. Here we describe the construction and validation of a vector (pPNT4) that considerably facilitates the generation of conditional gene-targeting constructs.

Cloning was started from the vectors pPNT (5) and p410, a vector containing the positive selection marker neomycin phosphotransferase flanked by frt sites (a kind gift by B. Luscher, Penn State University, PA, USA). The targeting vector pPNT comprises the neomycin phosphotransferase and thymidine kinase genes and the pBluescript ${ }^{\circledR}$ II SK $(+)$ backbone (Stratagene, La Jolla, CA, USA). As a first step, a BamHI linker (Oligo410-1a/b; 5'-GGGATCCGCGGCCGC-3'/5'-GCGGC-
CGCGGATCCC-3'; all linkers were purchased from ARK Scientific, Darmstadt, Germany) was inserted 3' from the frt-flanked neomycin phosphotransferase gene via SmaI into p410 to generate $\mathrm{p} 410.1$. Second, a LoxP/Not linker (Oligo410-2a/b; 5'-CGAAGCGGCCGCATAACTTCGTATAGCATA CATTATACGAAGTTAT-3' $/ 5^{\prime}$-CGATAACTTCGTATAATGTATGCTATACGAAGTTATGCGGCCGCTT-3'; the loxP sequence is underlined) was introduced $5^{\prime}$ of the frt-flanked neomycin phosphotransferase gene via BstBI to obtain p410.2. Third, OligopPNT1-a/ (5'-GGCCTAGGCCTCACTGGCCGTCGACTGTCTTCTGATCAGGCCTCACTGGCCGCGGCCGCTTCGAACCTGCA-3'/5'-GGTTCGAAGCGGCCGCGGCCAGTGAGGCCTGATCAGAAGACAGTCGACGGCCAGTGAGGCCTA-3') was inserted into pPNT digested with NotI and Sbfl to generate pPNT2. OligopPNT2-a/b (5'-GATCCGCTAGCGGTACCGAGCTCGAATTCATCGATCTCGAGA-3'/5'-AATTTCTCGAGATCGATGAATTCGAGCTCG-
GTACCGCTAGCG-3') harboring a set of single cutters (BamHI-NheI-KpnIEcoRI-ClaI-XhoI) was inserted into pPNT2 digested with BamHI and EcoRI to generate pPNT3. All linker cloning steps have been verified by sequencing. Finally, the frt-flanked neomycin phosphotransferase gene including the loxP site $5^{\prime}$ from the positive selection marker was transferred from $\mathrm{p} 410.2$ as a NotI/BamHI fragment into pPNT3 digested with NotI and BamHI to generate pPNT4 (Figure 1). The final vector pPNT4 shares with the parental pPNT vector the plasmid backbone and the negative selectable marker gene thymidine kinase. However, it contains a number of additional features: the neomycin phosphotransferase gene is flanked by frt sites, one loxP site is inserted $5^{\prime}$ of the neomycin resistance gene, and two multiple cloning sites are included for insertion of the two arms of the cellular gene for homologous recombination. With the appropriate mouse genomic sequence at hand, the final targeting vector is

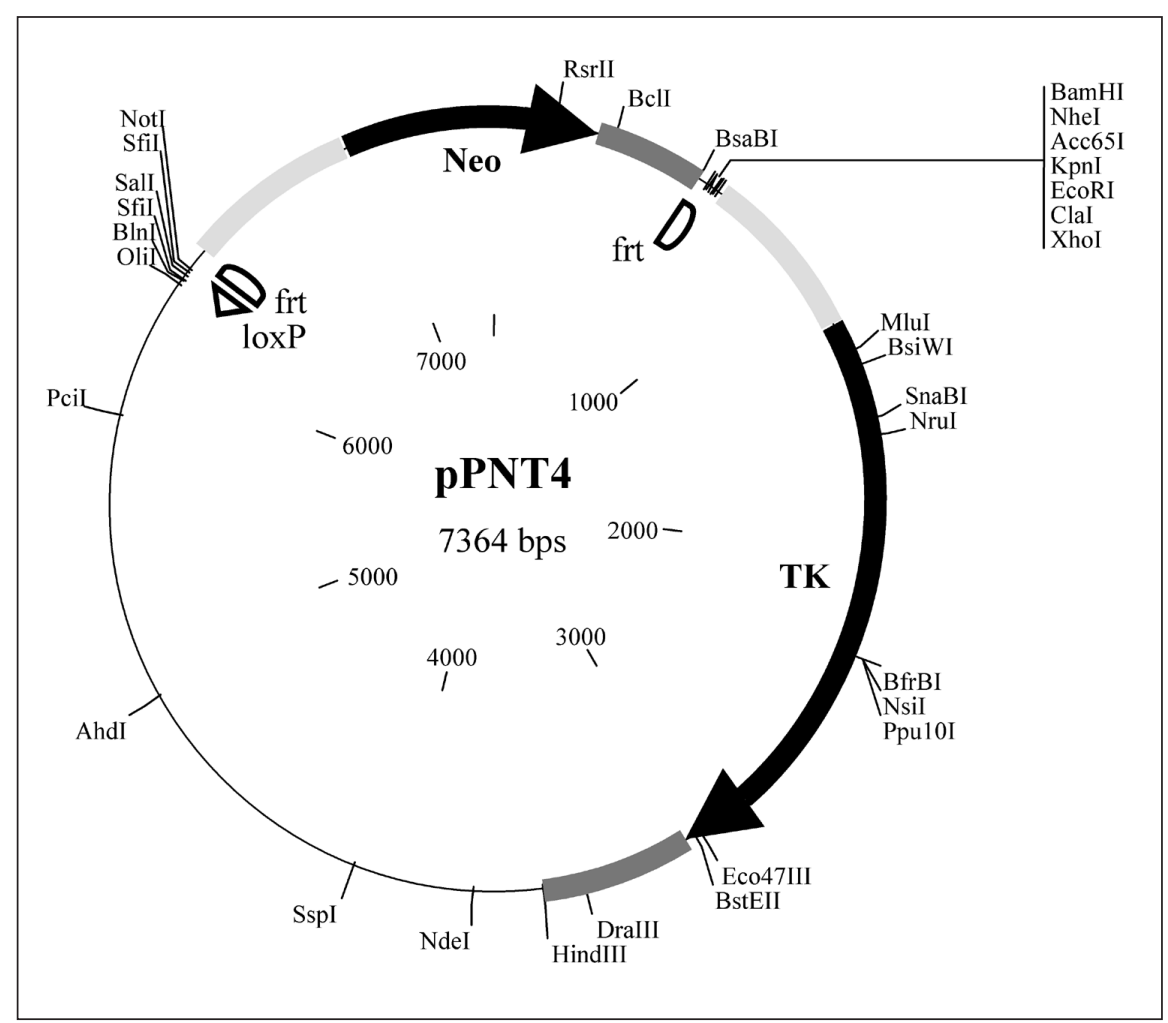

Figure 1. Schematics of the conditional gene targeting vector pPNT4. The promoter and polyadenylation regions of the PGK1 gene used for the expression of the neomycin phosphotransferase and the thymidine kinase genes are depicted in light and dark gray bars, respectively. The recognition sites for the Flp (frt) and Cre recombinase (loxP) are shown as half-circles or triangles, respectively. 


\section{Benchmarks}

generated by a minimum of three consecutive cloning steps: $(i)$ insertion of an additional lox $\mathrm{P}$ site, including at least one diagnostic restriction site in one of the arms of cellular DNA; and ( $i i$ and iii) sequential insertion of the two arms required for homologous recombination into the two multiple cloning sites of the targeting vector (Figure 2). If the second loxP site has been placed in the $3^{\prime}$ arm, then the neomycin resistance gene will be deleted by the Cre recombinase along with the gene of interest. If the second loxP site is inserted in the $5^{\prime}$ arm, then deletion of the cellular DNA fragment by Cre recombinase will leave the neomycin resistance gene untouched. In the latter case, removal of the neomycin resistance gene can be achieved by crossing the mice to Flpdeleter mice $(6,7)$. Importantly, the presence of the neomycin gene inside of an intron of the floxed cellular gene may impair the function of the gene of interest, thus creating a hypomorphic allele. A set of allelic variants may be created by either combining Cre and Flp mediated deletion or by separate deletion of the neomycin resistance gene and the floxed gene of interest. The vector is also suitable for the generation of constitutive conventional knock-out mice if the second loxP site is omitted and the removal of the resistance gene by Flp expression is desired.

Up to now, pPNT4 has been suc- cessfully used eight times in our laboratories as a targeting vector, three times for constitutive deletion of a gene, and five times for conditional gene targeting. Homologously recombined ES cell clones arose at a frequency of $1 \%-8 \%$ under standard ES cell conditions. Correct homologous recombination within the two arms was confirmed with both a $5^{\prime}$ and a $3^{\prime \prime}$ external probe located outside of the homologous arms (8). Germline transmission of the targeted alleles has been obtained in six cases up to now. Flpmediated removal of the frt-flanked neomycin phosphotransferase gene (neo) has been achieved in ES cells by transient expression of Flp recombinase from the plasmid pCAGGS-FLPe (9) with a frequency of $8 \%$ of the ES cell clones tested. The neomycin resistance gene has also been deleted in vivo by breeding mice harboring an frt-flanked neo gene with Flp-deleter mice $(6,7)$. Approximately $7 \%$ of the $\mathrm{F} 1$ offspring were positive for at least partial Flp-mediated neo removal. For germline transmission of the neodeleted allele, F1 mice were subsequently mated with $\mathrm{C} 57 \mathrm{BL} / 6$ mice. From this mating, $28 \%$ of the F2 offspring carried the neo-deleted allele. This is not surprising, as Flp-mediated neo removal in the $\mathrm{F} 1$ generation is incomplete; thus, less than $50 \%$ of the F2 offspring carry the neo-deleted al-

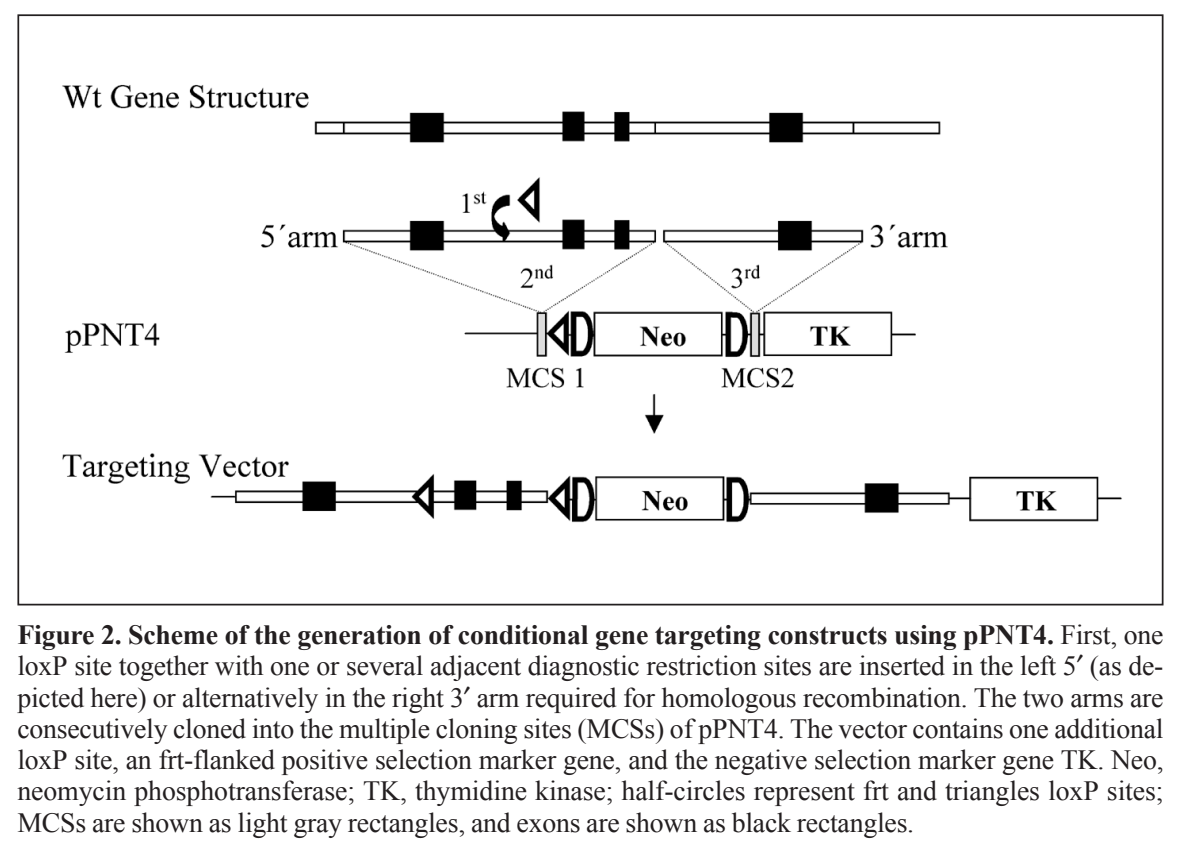

lele. Cre recombinase-mediated excision of the loxP-flanked (floxed) genomic fragment has also been obtained in vivo and worked with the expected frequency (10).

In summary, the described vector facilitates significantly the generation of constructs for conditional gene targeting. It has been shown to be suitable for gene targeting in ES cells and for the deletion of the gene of interest by Cre recombinase and of the neomycin resistance gene by Flp recombinase in vitro and in vivo.

\section{REFERENCES}

1.Waterston, R.H., K. Lindblad-Toh, E. Birney, J. Rogers, J.F. Abril, P. Agarwal, R. Agarwala, R. Ainscough, et al. 2002. Initial sequencing and comparative analysis of the mouse genome. Nature 420:520-562.

2.Fiering, S., C.G. Kim, E.M. Epner, and M. Groudine. 1993. An "in-out" strategy using gene targeting and FLP recombinase for the functional dissection of complex DNA regulatory elements: analysis of the $\beta$-globin locus control region. Proc. Natl. Acad. Sci. USA 90:8469-8473.

3.Kuhn, R., F. Schwenk, M. Aguet, and K. Rajewsky. 1995. Inducible gene targeting in mice. Science 269:1427-1429.

4.Sauer, B. and N. Henderson. 1988. Site-specific DNA recombination in mammalian cells by the Cre recombinase of bacteriophage P1. Proc. Natl. Acad. Sci. USA 85:5166-5170.

5.Tybulewicz, V.L., C.E. Crawford, P.K. Jackson, R.T. Bronson, and R.C. Mulligan. 1991. Neonatal lethality and lymphopenia in mice with a homozygous disruption of the cabl proto-oncogene. Cell 65:1153-1163.

6.Dymecki, S.M. 1996. Flp recombinase promotes site-specific DNA recombination in embryonic stem cells and transgenic mice. Proc. Natl. Acad. Sci. USA 93:6191-6196.

7.Rodriguez, C.I., F. Buchholz, J. Galloway, R. Sequerra, J. Kasper, R. Ayala, A.F. Stewart, and S.M. Dymecki. 2000. High-efficiency deleter mice show that FLPe is an alternative to Cre-loxP. Nat. Genet. 25:139140.

8.Joyner, A. L. 2000. Gene Targeting-A Practical Approach, 2nd ed. Oxford University Press, New York.

9.Schaft, J., R. Ashery-Padan, F. van der Hoeven, P. Gruss, and A.F. Stewart. 2001. Efficient FLP recombination in mouse ES cells and oocytes. Genesis 31:6-10.

10.Schwenk, F., U. Baron, and K. Rajewsky. 1995. A cre-transgenic mouse strain for the ubiquitous deletion of loxP-flanked gene segments including deletion in germ cells. Nucleic Acids Res. 23:5080-5081.

We thank R. Mulligan for providing the pPNT and B. Luscher for the 410 plas- 
mids. This work was supported by the Deutsche Forschungsgemeinschaft (Schwerpunktprogramm: Selenoproteine). Address correspondence to Dr. Marcus Conrad, Institute of Clinical Molecular Biology and Tumour Genetics, GSF Research Centre for Environment and Health, Marchioninistr. 25, D-81377, Munich, Germany. e-mail: marcus.conrad @gsf.de

Received 27 February 2003; accepted 2 April 2003.

Marcus Conrad, Markus
Brielmeier, Wolfgang Wurst,
and Georg W. Bornkamm
GSF Research Centre for
Environment and Health
Munich, Germany

Marcus Conrad, Markus and Georg W. Bornkamm GSF Research Centre for Munich, Germany

\section{Overcoming Obstacles in DNA Sequencing of Expression Plasmids for Short Interfering RNAs}

BioTechniques 34:1140-1144 (June 2003)

Gene silencing mediated by RNA interference (RNAi) has been observed in a wide range of eukaryotes including fungi, nematodes, insects, plants, and, more recently, mammalian cells. RNAi has been technologically developed to become a powerful tool to assess the function of genes. RNAi is induced by the presence of dsRNA whose sequence corresponds to a portion of the gene being targeted for silencing. The RNAi mechanism includes the degradation of dsRNA into short interfering RNAs (siRNAs) by Dicer and the degradation of the targeted RNA by RNA-induced silencing complex (RISC) (1-3).

One major barrier to the experimental use of RNAi for targeted gene silencing in mammalian systems was the nonspecific gene silencing triggered by long dsRNAs through the interferon response pathway (4). This difficulty was overcome when Tuschl and colleagues (5) discovered that siRNAs (21 bp), normally generated from long dsRNA during RNAi, can induce a specific and potent RNAi effect in cultured mammalian cells. Initially, siRNAs corresponding to the targeted genes were chemically synthesized in vitro and then were transfected into cultured mammalian cells, resulting in specific gene silencing. Soon thereafter, several different plasmids were designed to produce siRNAs in vivo from RNA transcripts generated by host cell RNA polymerases (6-11). These plasmids contain a promoter and terminator from genes transcribed by RNA polymerase III (such as the RNase P H1 RNA or U6 snRNA genes) so as to direct the expression of short RNAs with defined ends. Two different designs have been used for these siRNA-expressing vectors. In one strategy, the sense and antisense strands are expressed as two independent transcripts that anneal within the cell to form structures resembling in vitro synthesized siRNAs
$(7,8)$. In the second approach, the sense and antisense strands are expressed as a part of a single transcript separated by a short intervening loop. Therefore, the sense and antisense strands form a hairpin structure that after intracellular processing produces siRNAs $(6,9,10)$.

Since sequence complementarity between the siRNA and the targeted gene transcript is critical for efficient RNAi $(6,10,12)$, it becomes important to ensure that the siRNA expression plasmids contain no mutations introduced during the cloning process. However, siRNA vectors that express sense and antisense regions separated by an intervening loop will have hairpin secondary structures in the DNA that can interfere with standard sequencing reactions. Here we describe the use of digestion by restriction enzymes to relieve the secondary structure barriers to allow efficient DNA sequencing of inserts cloned into siRNA vectors.

We employed the pSUPER vector (6) to express siRNAs corresponding to the mammalian transcriptional coactivators BRG1 or p300 by cloning annealed oligonucleotides into the $B g l \mathrm{II}$ and HindIII sites of pSUPER as described (6). The BglII site was not restored after cloning. The sequence of the inserted double-stranded oligonucleotide for the BRG1 siRNA is indicated by the capital letters in Figure 1A.

The DNA sequences of the inserted fragments were analyzed using the BigDye $^{\mathrm{TM}}$ Terminator Cycle Sequencing Kit on a capillary-based ABI PRISM ${ }^{\circledR}$ 3100 Genetic Analyzer (both from Applied Biosystems, Foster City, CA, USA). Sequencing reactions on the two strands were initiated using primers corresponding to the T7 and T3 RNA polymerase promoters (5'-TAATACGACTCACTATAGGG- $3^{\prime}$ and 5' 5TTA- $^{\prime}$-ATTA ACCCTCACTAAAGGGA-3', respectively). Problematic sequencing data for both the BRG1 and p300 siRNA plasmids were consistently returned for sequencing reactions from either direction. These reads were typically accurate (Phred values $>20$ ) until encountering the inserted DNA oligonucleotides but then prematurely terminated 7-9 nucleotides into the siRNA encoding region (Figure 1B). A likely explanation for the premature termination of the sequencing reactions is the presence of 